\title{
Bioengineered NRF2-siRNA Is Effective to Interfere with NRF2 Pathways and Improve Chemosensitivity of Human Cancer Cells
}

\author{
Peng-Cheng Li, Mei-Juan Tu, Pui Yan Ho, Joseph L. Jilek, Zhijian Duan, Qian-Yu Zhang, Ai-Xi Yu, \\ and Ai-Ming Yu
}

Department of Orthopedics, Zhongnan Hospital of Wuhan University, Wuhan, Hubei, China (P.-C.L., A.-X.Y.) and Department of Biochemistry and Molecular Medicine, Comprehensive Cancer Center, UC Davis School of Medicine, Sacramento, California (P.-C.L., M.-J.T., P.Y.H., J.L.J., Z.D., Q.-Y.Z., A.-M.Y.)

Received September 22, 2017; accepted October 18, 2017

\section{ABSTRACT}

The nuclear factor (erythroid-derived 2)-like 2 (NRF2) is a transcription factor in the regulation of many oxidative enzymes and efflux transporters critical for oxidative stress and cellular defense against xenobiotics. NRF2 is dysregulated in patient osteosarcoma (OS) tissues and correlates with therapeutic outcomes. Nevertheless, research on the NRF2 regulatory pathways and its potential as a therapeutic target is limited to the use of synthetic small interfering RNA (siRNA) carrying extensive artificial modifications. Herein, we report successful high-level expression of recombinant siRNA against NRF2 in Escherichia coli using our newly established noncoding RNA bioengineering technology, which was purified to $>99 \%$ homogeneity using an anion-exchange fast protein liquid chromatography method. Bioengineered NRF2-siRNA was able to significantly knock down NRF2 mRNA and protein levels in human OS 143B and MG63 cells, and subsequently suppressed the expression of NRF2-regulated oxidative enzymes [heme oxygenase-1 and $N A D(P) H: q u i n o n e$ oxidoreductase 1] and elevated intracellular levels of reactive oxygen species. In addition, recombinant NRF2siRNA was effective to sensitize both 143B and MG63 cells to doxorubicin, cisplatin, and sorafenib, which was associated with significant downregulation of NRF2-targeted ATP-binding cassette ( $A B C$ ) efflux transporters ( $A B C C 3, A B C C 4$, and $A B C G 2$ ). These findings support that targeting NRF2 signaling pathways may improve the sensitivity of cancer cells to chemotherapy, and bioengineered siRNA molecules should be added to current tools for related research.

\section{Introduction}

The nuclear factor (erythroid-derived 2)-like 2 (NRF2 or NFE2L2) is a transcription factor that plays a pivotal role in cellular defense against oxidative and electrophilic stressors or xenobiotics via the induction of a number of oxidative [e.g., heme oxygenase-1 (HO-1) and $\mathrm{NAD}(\mathrm{P}) \mathrm{H}$ : quinone oxidoreductase 1 (NQO1)] and conjugating enzymes (e.g., glutathione $S$-transferases), as well as efflux ATP-binding cassette (ABC) transporters (e.g., ABCC4 and ABCG2) [see review by Furfaro et al. (2016)]. Therefore, activation of NRF2 is traditionally considered as a chemopreventive pathway in xenobiotic metabolism and disposition, and NRF2 has been regarded as a tumor suppressor in cancer research fields. However, there is accumulating evidence that NRF2 may be overactivated in cancer cells, which creates an environment for promoting the survival of cancer cells and conferring resistance to chemotherapy (Jaramillo and

This work was supported in part by Outstanding Medical Academic leader Program of Hubei Province and Huanghe Yingcai Program of Wuhan (A.-X.Y.) and the National Institutes of Health [Grants R01GM113888 and U01CA175315 (A.-M.Y.)]. P.-C. L. was supported by a Graduate Student Fellowship from the Academic Exchange Program at Wuhan University. J.L.J. was supported by a NIGMS-funded Pharmacology Training Program Grant [T32GM099608].

https://.doi.org/10.1124/dmd.117.078741.
Zhang, 2013; Huang et al., 2015; Furfaro et al., 2016). Therefore, more studies are expected to advance our understanding of NRF2 regulatory pathways in cancer cells toward improved cancer therapies.

Osteosarcoma (OS) is the most common primary sarcoma of bone among children, adolescents, and young adults (Rivera-Valentin et al., 2015). Anatomically, OS is localized mainly in the femur (40\%), tibia (20\%), and humerus (10\%). A major strategy for the treatment of OS patients is the use of neoadjuvant chemotherapy and subsequent extensive resection of tumor tissues followed by further adjuvant chemotherapy for high-grade lesions, which includes high doses of methotrexate, doxorubicin, and cisplatin (sometimes with ifosfamide) (Jaffe, 2009; Kansara et al., 2014). While this regimen helps OS patients avoid amputation and may dramatically improve overall survival, the 5 -year survival rate is around $70 \%$. Moreover, OS exhibits a predilection for pulmonary metastases, and the 5-year survival rate for OS patients with lung metastases drops sharply to $18 \%-33 \%$ (Luetke et al., 2014). Therefore, there is a clear need for the development of new therapeutic strategies to fight malignant OS.

The potential roles of NRF2 in human OS cells have been demonstrated by some studies. First, there is an increased expression of NRF2 among OS patient specimens that is associated with poor clinical outcome and disease-free survival (Park et al., 2012; Zhang et al., 2016). Second, NRF2 expression levels are higher in human OS

ABBREVIATIONS: ABC, ATP-binding cassette; ANOVA, analysis of variance; BERA, bioengineered RNAi agent; FPLC, fast protein liquid chromatography; HO-1, heme oxygenase-1; KEAP1, Kelch-like ECH-associated protein 1; miR or miRNA, microRNA; MSA, sephadex aptamertagged methionyl-tRNA; ncRNA, noncoding RNA; NQO1, NAD(P)H:quinone oxidoreductase 1; NRF2, nuclear factor (erythroid-derived 2)-like 2; OS, osteosarcoma; qPCR, quantitative polymerase chain reaction; RNAi, RNA interference; ROS, reactive oxygen species; siRNA, small interfering RNA; shRNA, short hairpin RNA; tRNA, transfer RNA. 
cells following $\alpha$-radiation, which may be attenuated by short hairpin RNA (shRNA)-mediated gene silencing toward a higher sensitivity to radiation (Chen et al., 2017). On the other hand, lower protein levels of NRF2 and its target HO-1 are accompanied by the increased sensitivity of OS cells to $\gamma$-irradiation after the interference of DNA-dependent protein kinase catalytic subunit (Tang et al., 2014). Furthermore, pharmacological and genetic interference of the prosurvival p38 MAPK/AKT/NRF2/EGR1/HO-1 axis sensitizes OS cells to 15-deoxy$\Delta$-12,14-prostaglandin J2-mediated apoptosis (Koyani et al., 2016).

Although NRF2 represents a promising therapeutic target for the treatment of cancer, current RNA interference (RNAi) materials are limited to synthetic small interfering RNAs (siRNAs) or shRNAexpressing agents (Singh et al., 2008; Fourtounis et al., 2012; Lu et al., 2016), in addition to small-molecule modulators that might not be very potent or selective (Sun et al., 2017). Utilization of shRNA-expressing agents (e.g., viral or nonviral vector-based systems) may complicate RNAi processes because they are literally DNA materials that rely on not only the efficiency of gene delivery but also the capacity of host cells or organisms in transcribing DNA to noncoding RNAs (ncRNAs) before the execution of siRNA actions. Furthermore, chemically engineered siRNAs are composed of excessive artificial modifications that may exhibit distinct structures, physiochemical properties, biologic activities, and safety profiles from natural RNAs produced in cells. Therefore, we recently developed a novel ncRNA bioengineering technology for the production of natural RNAi agents [e.g., microRNAs (miRNAs) and
siRNAs] (Li et al., 2014, 2015; Chen et al., 2015; Wang et al., 2015). In particular, the transfer RNA (tRNA)/pre-miR-34a is a robust and versatile carrier to accommodate target small RNAs (Fig. 1A) and offers high-level expression of recombinant ncRNAs (Chen et al., 2015). Folded within cells and without artificial modification, these bioengineered RNAi agents (BERAs) may better capture the stability, activity, and safety profiles of natural RNAs, and thus represent a new class of RNAi materials for biomedical research (Duan and $\mathrm{Yu}, 2016$; Ho and $\mathrm{Yu}, 2016)$.

In this study, we aimed to produce a BERA against NRF2 (BERA/NRF2-siRNA) and to assess the application of bioengineered NRF2-siRNA to interfering NRF2 pathways in human OS cells. Following the successful high-level expression of BERA/NRF2-siRNA molecules in Escherichia coli using this novel tRNA/pre-miR-34a-based ncRNA bioengineering approach (Chen et al., 2015), recombinant BERA/NRF2-siRNA was purified to $>99 \%$ homogeneity by an anionexchange fast protein liquid chromatography (FPLC) method. We further demonstrated that BERA/NRF2-siRNA was selectively processed to target siRNA in human OS cells and remarkably knocked down NRF2 mRNA and protein levels, as compared with cells treated with vehicle or the scaffold sephadex aptamer-tagged methionyl-tRNA (MSA), which has been demonstrated as a valid control for assessing the actions of BERAs through various RNA sequencing and functional studies (Chen et al., 2015; Li et al., 2015; Wang et al., 2015). In addition, a number of NRF2-regulated oxidative enzymes and efflux
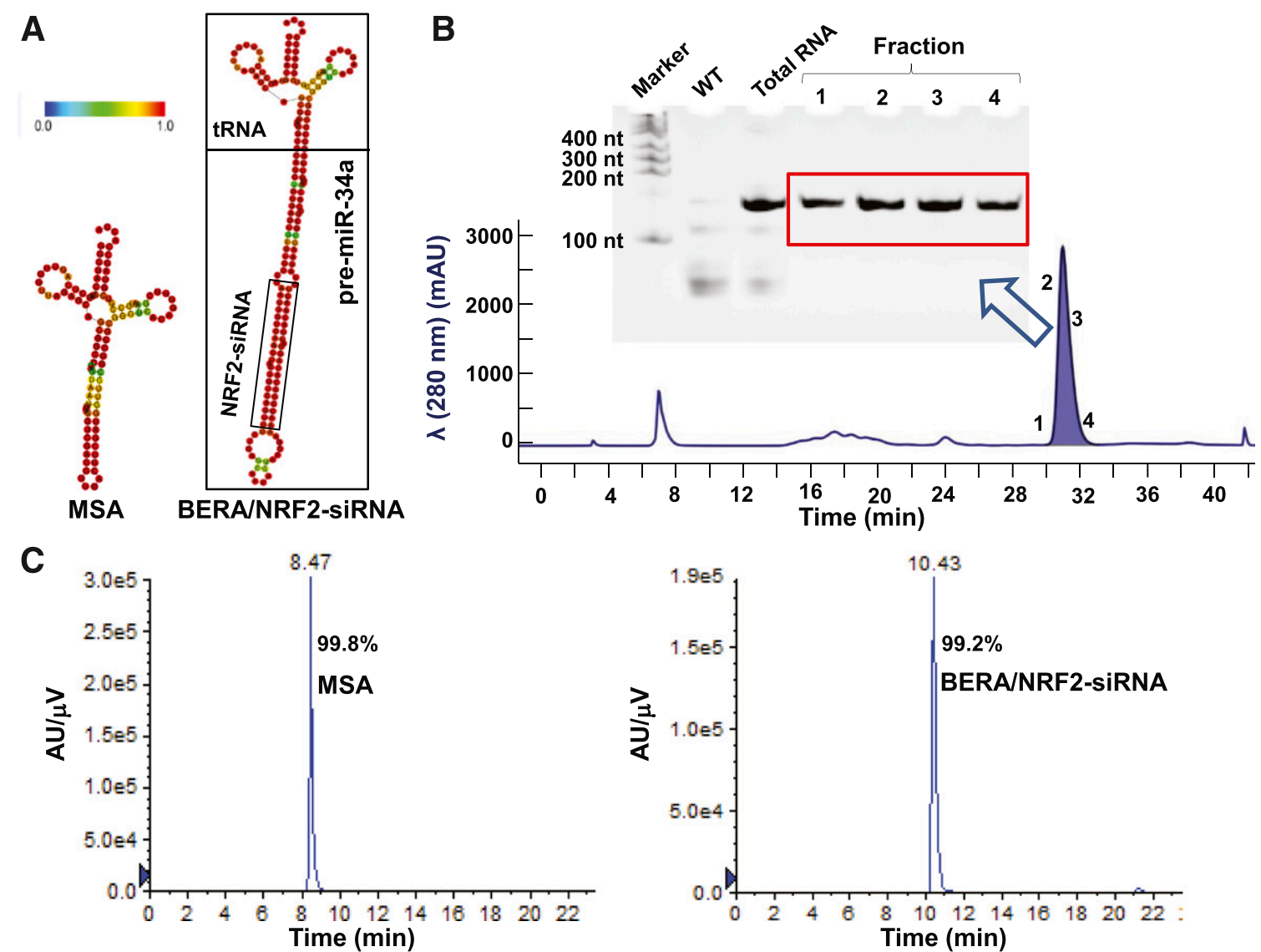

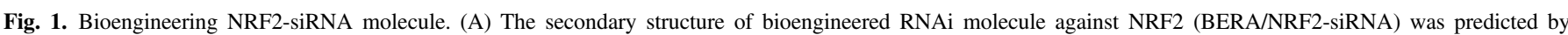

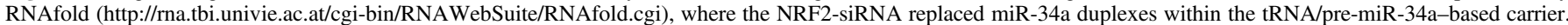

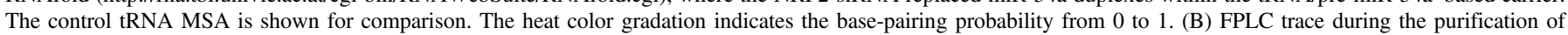

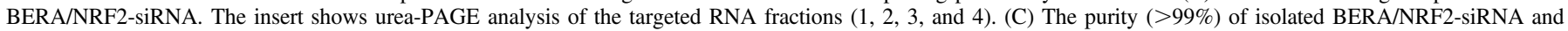
control MSA was confirmed by high-performance liquid chromatography analysis. nt, nucleotide; WT, wild type. 
ABC transporters were subsequently downregulated in OS cells, leading to higher levels of intracellular reactive oxygen species (ROS) and chemosensitivity.

\section{Materials and Methods}

Chemicals and Materials. Doxorubicin and sorafenib were purchased from LC Laboratories (Woburn, MA). Cisplatin, protease inhibitor cocktail, and Trizol reagent were bought from Sigma-Aldrich (St. Louis, MO). Thiazolyl blue tetrazolium bromide, dimethylsulfoxide, and bovine serum albumin were bought from VWR (Radnor, PA). RPMI 1640 medium, fetal bovine serum, 0.05\% trypsin-EDTA, radioimmunoprecipitation buffer, BCA Protein Assay Kit, and Lipofectamine 3000 were purchased from Thermo Fisher Scientific (Waltham, MA). Polyvinylidene fluoride membrane, blotting-grade blocker, and Western ECL Substrate kit were purchased from Bio-Rad (Hercules, CA). Direct-zol RNA MiniPrep kit was bought from Zymo Research (Irvine, CA). All other chemicals and organic solvents of analytical grade were purchased from Thermo Fisher Scientific or Sigma-Aldrich.

Expression and FPLC Purification of BERA/NRF2-siRNA. The production of BERA/NRF2-siRNA and control MSA was carried out using the ncRNA bioengineering technology as we described recently (Li et al., 2014, 2015; Chen et al., 2015). In brief, a DNA fragment encoding the NRF2-siRNA (5'-UAAUUGUCAACUACUGUCAGUU- ${ }^{\prime}$ ) and complementary sequence was cloned into pBSTNAV linearized by SacII and EagI (New England Biolabs, Ipswich, MA). Plasmids were amplified in the DH5 $\alpha$ strain and confirmed by sequencing analyses (Genscript, Piscataway, NJ). Recombinant ncRNA was produced in HST08 E. coli and verified by denaturing urea $(8 \mathrm{M})$ polyacrylamide (8\%) gel electrophoresis (PAGE) analysis of total bacterial RNA isolated by phenol extraction.

Anion-exchange FPLC purification of BERA/NRF2-siRNA was conducted on an NGC QUEST 10PLUS FPLC system (Bio-Rad) consisting of a fraction collector ( $\mathrm{Li}$ et al., 2014; Chen et al., 2015). Following urea-PAGE analysis, FPLC fractions containing pure target RNAs were pooled. Recombinant ncRNAs were precipitated with ethanol, reconstituted with nuclease-free water, desalted, and then concentrated with Amicon ultra 0.5-ml centrifugal filters (30 KD; EMD Millipore, Billerica, MA). RNA concentrations were determined with a NanoDrop 2000 Spectrophotometer (Thermo Fisher Scientific), and RNA purity (Fig. 1) was further determined by a high-performance liquid chromatography assay (Chen et al., 2015; Wang et al., 2015).

Cell Culture and Transfection. Human OS cell lines 143B (CRL-8303) and MG63 (CRL-1543) were purchased from the American Type Culture Collection (Manassas, VA) with satisfactory authentication. Cells were maintained in RPMI 1640 medium containing $10 \%$ fetal bovine serum at $37^{\circ} \mathrm{C}$ in a humidified atmosphere with 5\% carbon dioxide and 95\% air. Upon arrival, cell lines were immediately expanded and frozen. Cell lines used in experiments were replaced with these cryopreserved stocks after 8-12 passages. The BERA/NRF2-siRNA and control MSA were transfected into the human OS cells within the logarithmic growth phases by using Lipofectamine 3000 reagent, according to the manufacturer's instructions.

RNA Isolation and Reverse-Transcription Quantitative Real-Time Polymerase Chain Reaction. Human OS 143B and MG63 cells were treated with $10 \mathrm{nM}$ BERA/NRF2-siRNA or MSA for 48 hours. Total RNAs were isolated with the Direct-zol RNA MiniPrep kit. cDNA was synthesized from total RNAs using NxGen M-MuLV reverse transcriptase (Lucigen, Middleton, WI) with random hexamers or respective stem-loop primers (Table 1). Quantitative polymerase chain reaction (qPCR) analyses were carried out using quantitative real-time PCR Green Supermix (Bio-Rad) and gene-specific primers (Table 1) on a CFX96 Touch real-time PCR system (Bio-Rad), as described previously (Chen et al., 2015; Zhao et al., 2015, 2016). Glyceraldehyde-3-phosphate dehydrogenase and U6 were used as the internal control for the quantification of mRNA and siRNA levels, respectively. Cells were treated in triplicate and assayed separately. The comparative threshold cycle $(\mathrm{Ct})$ method with the eq. $2^{-\Delta \Delta \mathrm{Ct}}$ was used to calculate the relative gene expression.

Protein Isolation and Western Blots. Human OS 143B and MG63 cells were treated with $10 \mathrm{nM}$ BERA/NRF2-siRNA or MSA for 48 hours. Proteins were extracted from harvested cells using radioimmunoprecipitation lysis buffer supplemented with complete protease inhibitors, and protein concentrations were determined using the BCA Protein Assay Kit. The whole-cell proteins
TABLE 1

The sequences of primers used in the study

\begin{tabular}{|c|c|}
\hline Gene & Sequence \\
\hline \multicolumn{2}{|l|}{ U6 } \\
\hline Forward & $5^{\prime}$-CTCGCTTCGGCAGCACA-3' \\
\hline Reverse & 5'-AACGCTTCACGAATTTGCGT-3' \\
\hline \multicolumn{2}{|l|}{ NRF2-siRNA } \\
\hline RT & $\begin{array}{l}5^{\prime} \text {-GTCGTATCCAGTGCAGGGTCCGAGG } \\
\text { TATTCGCACTGGATACGACAACTGA-3' }\end{array}$ \\
\hline Forward & 5'-GGCGCCTAATTGTCAACTTCTG-3' \\
\hline Reverse & 5'-GTGCAGGGTCCGAGGT-3' \\
\hline \multicolumn{2}{|c|}{ BERA/NRF2-siRNA } \\
\hline Forward & 5'-GGCCAGCTGTGAGTGTTTCTT-3' \\
\hline Reverse & $5^{\prime}$-GGGCCAACAACGTGCAGC-3' \\
\hline \multicolumn{2}{|l|}{ GAPDH } \\
\hline Forward & 5'-ATCACCATCTTCCAGGAGCGA-3' \\
\hline Reverse & 5'-GCTTCACCACCTTCTTGATGT-3' \\
\hline \multicolumn{2}{|l|}{ NRF2 } \\
\hline Forward & 5'-TGAGGTTTCTTCGGCTACGTT-3' \\
\hline Reverse & 5'-CTTCTGTCAGTTTGGCTTCTGG-3' \\
\hline \multicolumn{2}{|l|}{ HO-1 } \\
\hline Forward & 5'-CTGGAGGAGGAGATTGAGCG-3' \\
\hline Reverse & 5'-ATGGCTGGTGTGTAGGGGAT-3' \\
\hline \multicolumn{2}{|l|}{ NQO1 } \\
\hline Forward & 5'- TGCAGCGGCTTTGAAGAAGAAAGG-3' \\
\hline Reverse & 5'-TCGGCAGGATACTGAAAGTTCGCA- $3^{\prime}$ \\
\hline \multicolumn{2}{|l|}{$\mathrm{ABCC} 1$} \\
\hline Forward & 5'-AACCTGGACCCATTCAGCC-3' \\
\hline Reverse & 5'-GACTGGATGAGGTCGTCCGT-3' \\
\hline \multicolumn{2}{|l|}{$\mathrm{ABCC} 2$} \\
\hline Forward & 5'-AGCAGCCATAGAGCTGGCCCT-3' \\
\hline Reverse & 5'-AGCAAAACCAGGAGCCATGTG-3' \\
\hline \multicolumn{2}{|l|}{$\mathrm{ABCC} 3$} \\
\hline Forward & 5'-CAGAGAAGGTGCAGGTGACA-3' \\
\hline Reverse & 5'-CTAAAGCAGCATAGACGCCC-3' \\
\hline \multicolumn{2}{|l|}{$\mathrm{ABCC} 4$} \\
\hline Forward & 5'-TGATGAGCCGTATGTTTTGC-3' \\
\hline Reverse & 5'-CTTCGGAACGGACTTGACAT-3' \\
\hline \multicolumn{2}{|l|}{ ABCG2 } \\
\hline Forward & 5'-CAGGTGGAGGCAAATCTTCGT-3' \\
\hline Reverse & $5^{\prime}$-ACACACCACGGATAAACTGA- $3^{\prime}$ \\
\hline
\end{tabular}

GAPDH, glyceraldehyde-3-phosphate dehydrogenase; RT, reverse transcription.

(35 $\mu \mathrm{g} /$ lane) were resolved on a $10 \%$ SDS-PAGE gel and transferred to a polyvinylidene fluoride membrane. Membranes were incubated in 5\% BlottingGrade Blocker (catalog \#170-6404; Bio-Rad) at room temperature for 2 hours, and then with primary antibodies, anti-NRF2 antibody (1:1000 dilution, catalog \#ab62352; Abcam, Cambridge, MA), or anti- $\beta$-actin antibody (1:5000 dilution, catalog \#A5441; Sigma-Aldrich) overnight at $4^{\circ} \mathrm{C}$. The membranes were subsequently incubated with the secondary horseradish peroxidase-labeled antimouse (1:3000 dilution, catalog \#7076; Cell Signaling Technology, Danvers, MA) or anti-rabbit IgG antibodies (1:10,000 dilution, catalog \#111035003); Jackson ImmunoResearch, West Grove, PA) for 2 hours, followed with Clarity Western ECL substrates. Proteins were visualized and acquired with a ChemiDoc MP Imaging System (Bio-Rad) and thus quantified by Image Laboratory software (Bio-Rad). NRF2 protein levels were normalized to $\beta$-actin levels in corresponding samples for comparison.

Reactive Oxygen Species Assay. The intracellular ROS levels were determined using the Fluorometric Intracellular ROS kit (Sigma-Aldrich), according to the manufacturer's instructions. In brief, cells were treated with $10 \mathrm{nM}$ BERA/NRF2-siRNA, MSA, or vehicle control for 48 hours. Fluorescence intensity was recorded (excitation $520 \mathrm{~nm}$ and emission $605 \mathrm{~nm}$ ) using a SpectraMax microplate reader (Molecular Devices, Sunnyvale, CA), and ROS levels were normalized to the vehicle control treatment group, which was set as $100 \%$.

Cytotoxicity Assay, Chemosensitivity, and Dose-Response Relationship. Cells were seeded in a 96-well plate (Corning Inc., Corning, NY) at $5 \times 10^{3} /$ well in $200 \mu \mathrm{l}$ of medium and incubated at $37^{\circ} \mathrm{C}$ overnight. To determine the cytotoxicity of BERA/NRF2-siRNA, cells were treated with different concentrations $(0-40 \mathrm{nM})$ of BERA/NRF2-siRNA or MSA. To evaluate the impact of BERA/NRF2-siRNA on chemosensitivity, cells were treated with $2 \mathrm{nM}$ and/or 
BERA/NRF2-siRNA or MSA plus various concentrations of doxorubicin (0-300 nM), cisplatin (0-3000 nM), or sorafenib (0-3000 nM). After 48 hours, cell viability was determined using thiazolyl blue tetrazolium bromide assay, as we described previously (Wang et al., 2015; Zhao et al., 2015, 2016).

Cell viability data were normalized to corresponding vehicle control (0 nM drug), which was set as $100 \%$. An inhibitory, normalized response model with variable slope $\left[\mathrm{Y}=100 /\left(1+10^{\wedge}\left(\left(\operatorname{LogIC} \mathrm{IC}_{50}-\mathrm{X}\right) \times\right.\right.\right.$ Hill slope $\left.)\right)$; GraphPad Prism, San Diego, CA] was used to estimate the pharmacodynamic parameters (EC $\mathrm{E}_{50}$ and Hill slope) for the antiproliferative activities of BERA/NRF2-siRNA and MSA since the ncRNAs at the tested concentrations showed a full inhibition. By contrast, chemosensitivity data were fitted to an inhibitory dose-response model with variable slope $\left[\mathrm{Y}=\right.$ bottom $+($ top - bottom $) /\left(1+10^{\wedge}\left(\left(\operatorname{LogIC}{ }_{50}-\mathrm{X}\right) \times\right.\right.$ Hill slope)); "top" was not constrained, whereas "bottom" was constrained to 0 because a low concentration of BERA/NRF2-siRNA in the absence of chemotherapeutics already inhibited cell proliferation to certain degrees, and this model offered an acceptable Goodness of fit $\left.\left(R^{2}=0.80-0.99\right)\right]$.

Statistical Analysis. All values were presented as the mean \pm S.D. as experiments were carried out in triplicate independently. Data were analyzed using one-way or two-way analysis of variance (ANOVA) or Student's $t$ test (GraphPad Prism), depending upon the numbers of groups and variances. Difference was considered as statistically significant when the probability was less than $0.05(P<0.05)$

\section{Results}

Expression of BERA/NRF2-siRNA in $E$. coli and Purification to a High Homogeneity. To produce recombinant NRF2-siRNA agent in E. coli, we used the tRNA/pre-miR-34a-based ncRNA bioengineering technology (Chen et al., 2015), where miR-34a duplexes were substituted by target NRF2-siRNA (Fig. 1A). The predicted secondary structure of BERA/NRF2-siRNA appeared to maintain the stable premiR-34a hairpin structure as well as tRNA D-loop and T-loop structures that are identical to MSA. To evaluate the expression of BERA/NRF2siRNA in E. coli, total RNA was isolated and subjected to urea-PAGE analysis. The appearance of a new strong RNA band at the expected size (Fig. 1B) in bacteria transformed with BERA/NRF2-siRNA expression plasmid, as compared with the wild-type bacteria, indicated a successful high-level expression of recombinant BERA/NRF2-siRNA in E. coli.

To purify BERA/NRF2-siRNA, total RNAs were isolated from bacteria and separated by an anion-exchange FPLC method (Chen et al., 2015; Wang et al., 2015). With real-time UV monitoring, FPLC fractions were collected when the peak of target RNA appeared (Fig. 1B). A high degree of homogeneity was verified by urea-PAGE analysis of individual fractions, which were combined and desalted to offer final BERA/NRF2-siRNA products. Further high-performance liquid chromatography analysis revealed that the resultant BERA/NRF2-siRNA was highly homogeneous ( $>99 \%$ pure; Fig. 1C). Given a high-level expression, we generally obtained $18-25 \mathrm{mg}$ of total RNAs from $1 \mathrm{~L}$ of bacterial culture, which offered 4-6 mg of pure BERA/NRF2-siRNA following FPLC purification (equivalent to $\sim 20 \%$ yield of recombinant BERA/NRF2-siRNA/total RNAs). Likewise, the control scaffold tRNA MSA for assessing the functions of bioengineered RNAi agents, whose validity has been demonstrated by RNA sequencing studies as well as functional analyses (Li et al., 2014; Chen et al., 2015; Wang et al., 2015), was expressed in E. coli and purified to a high degree of homogeneity (99.8\% pure; Fig. 1C).

BERA/NRF2-siRNA Is Processed to NRF2-siRNA in Human OS Cells and Effectively Knocks Down NRF2 Levels. To examine if BERA/NRF2-siRNA could be processed to target NRF2-siRNA in human OS 143B and MG63 cells, regular qPCR and stem-loop real-time qPCR were conducted to quantitate NRF2-siRNA precursor and NRF2siRNA, respectively, in cells treated with recombinant ncRNAs for 48 hours. The data showed that NRF2-siRNA precursor and NRF2-siRNA levels were approximately 4-fold and 20-fold higher, respectively, in 143B cells transfected with BERA/NRF2-siRNA than MSA or vehicle control (Fig. 2, A and B). Similarly, BERA/NRF2-siRNA-treated MG63 cells showed 30-fold and 8-fold higher levels of NRF2-siRNA precursor and NRF2-siRNA, respectively, than MSA or vehicle control. These results demonstrated that BERA/NRF2-siRNA was successfully transfected into human OS cells and processed to target NRF2-siRNA.

To determine the impact of bioengineered NRF2-siRNA on NRF2 expression in human OS cells, qPCR and immunoblots were carried out to examine NRF2 mRNA and protein levels with specific primers and antibody, respectively. Whereas low concentrations of MSA (10 nM) showed no or minimal effects on NRF2 mRNA or protein expression, as compared with vehicle control (Fig. 2, C and D), BERA/NRF2-siRNA knocked down NRF2 mRNA levels by approximately $70 \%$ and $60 \%$ in 143B and MG63 cells, respectively, compared with either MSA or vehicle treatment (Fig. 2C). Furthermore, as reported in other types of human carcinoma cell lines (Lau et al., 2013), endogenous NRF2 proteins expressed in human OS cells showed two bands that migrate at around $110 \mathrm{kD}$ instead of the predicted molecular mass of $55-65 \mathrm{kD}$ (Fig. 2D). The upper NRF2 bands $(\sim 110 \mathrm{kD})$ in 143B and MG63 cells were almost completely knocked out by BERA/NRF2-siRNA, whereas the lower bands ( $~ 95 \mathrm{kD}$ ) showed 20\%-30\% suppression, as compared with MSA or vehicle control. When the densities of two bands were combined together, NRF2 protein levels were reduced by $60 \%$ in 143B cells and 70\% in MG63 cells, respectively, by BERA/NRF2siRNA (Fig. 2D). These findings indicated that bioengineered NRF2siRNA was effective to silence NRF2 mRNA and protein expression within human OS cells.

Bioengineered NRF2-siRNA Reduces the Expression of NRF2Regulated Oxidative Enzymes in Human OS Cells and Alters Intracellular ROS Levels. Since NRF2 is a major regulator of genes encoding oxidative enzymes in response to oxidative stress (Furfaro et al., 2016), we examined the consequent effects of bioengineered NRF2-siRNA on HO-1 and NQO1 gene expression as well as cellular ROS accumulation. As determined by qPCR analyses, silencing NRF2 by BERA/NRF2-siRNA (Fig. 2) led to a $50 \%$ and $60 \%$ downregulation of HO-1 and NQO1 mRNA levels, respectively, in 143B cells (Fig. 3, A and B) compared with either MSA or vehicle treatment. Subsequently, a $25 \%$ increase of intracellular ROS levels was identified in 143B cells treated with recombinant NRF2-siRNA, as compared with the same doses of MSA (10 nM) or vehicle controls (Fig. 3C). Likewise, ROS levels were significantly higher in BERA/NRF2-siRNA-treated MG63 cells, as a result of the downregulation of HO-1 and NQO1 (Fig. 3), supporting the actions of bioengineered NRF2-siRNA on NRF2controlled ROS detoxification.

Biologic NRF2-siRNA Is Pharmacologically Active to Inhibit OS Cell Proliferation. We further defined the antiproliferative activity of bioengineered NRF2-siRNA following the interference of the NRF2 signaling pathway. Our data showed that BERA/NRF2siRNA reduced the proliferation of both 143B and MG63 cells in a dose-dependent manner and, most importantly, to a significantly greater degree than MSA control $(P<0.001$, two-way ANOVA) (Fig. 4A), despite the fact that high doses of MSA were also able to inhibit cell proliferation ( $\mathrm{Li}$ et al., 2015; Wang et al., 2015). The effectiveness of BERA/NRF2-siRNA in suppressing human OS 143B and MG63 cell growth was further manifested by the calculated $\mathrm{EC}_{50}$ values, which were much lower than the MSA control (Fig. 4B). Together, these findings revealed that bioengineered NRF2-siRNA was biologically or pharmacologically active in the modulation of OS cell survival, which may be at least partially attributable to the perturbation of NRF2-regulated ROS signaling (Fig. 3). 

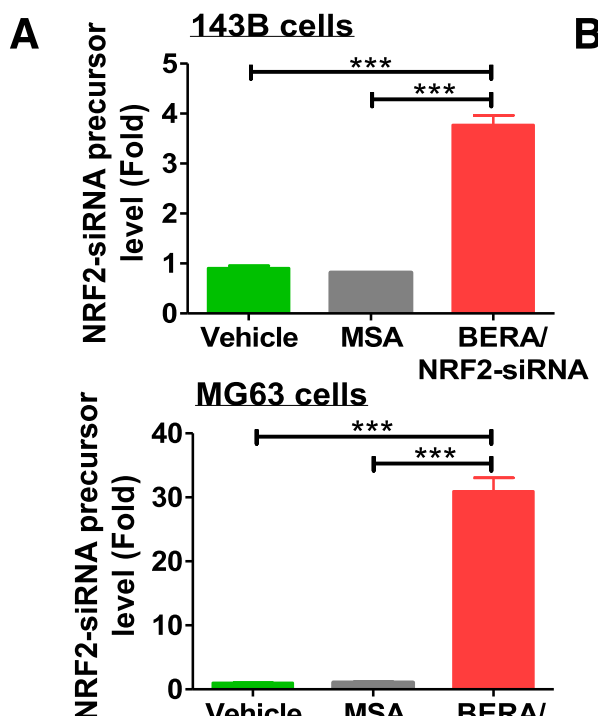

B

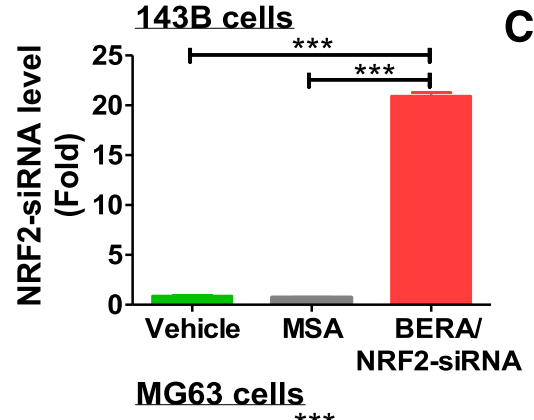

C
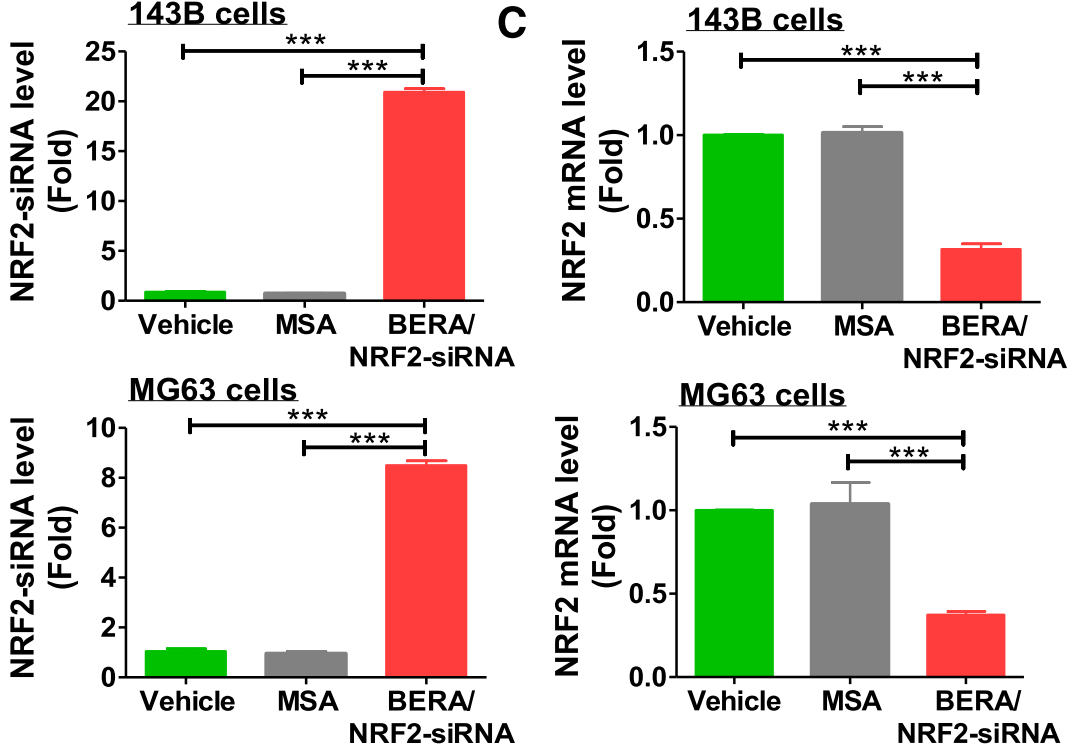

D

\section{BERA/}

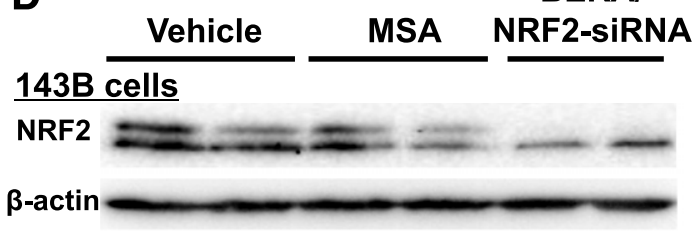

MG63 cells NRF2

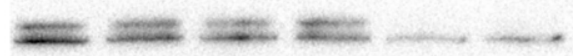

$\beta$-actin
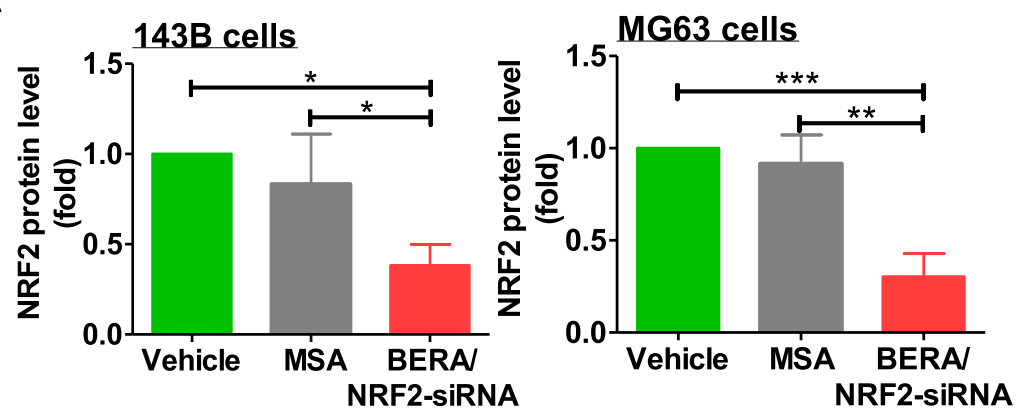

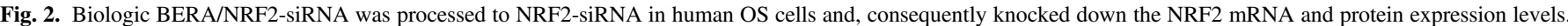

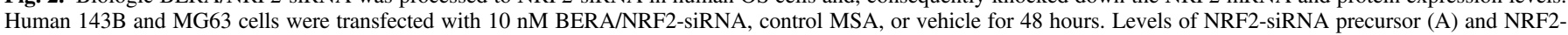

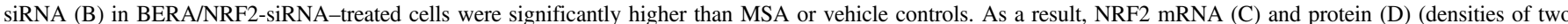

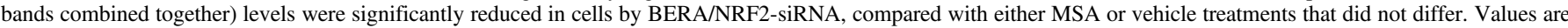
the mean \pm S.D. of triplicate treatments. $* P<0.05 ; * * P<0.01$; ***P<0.001, compared with vehicle or MSA control group (one-way ANOVA).

Bioengineered NRF2-siRNA Modulates the Expression of NRF2Regulated ABC Transporters in Human OS Cells. Recent studies have also demonstrated the role of NRF2 in the regulation of efflux ABC transporters (Adachi et al., 2007; Aleksunes et al., 2008; Malhotra et al., 2010; Singh et al., 2010; Chorley et al., 2012; Canet et al., 2015; Francois et al., 2017). Therefore, we assessed the influence of BERA/NRF2-siRNA on the mRNA levels of five ABC transporters, ABCC1-4/MRP1-4 and ABCG2/breast cancer resistance protein, in human OS cells. Our data showed that, compared with vehicle control, $10 \mathrm{nM}$ MSA did not alter the expression of ABC transporters examined (Fig. 5). Interestingly, only ABCC3 mRNA levels were significantly reduced by BERA/NRF2-siRNA in 143B cells by approximately $55 \%$, as compared with either MSA or vehicle treatment, whereas other ABC transporters were not altered (Fig. 5A). By contrast, BERA/NRF2siRNA suppressed ABCC4 and ABCG2 levels by $30 \%$ and $25 \%$, respectively, in MG63 cells, compared with MSA or vehicle controls (Fig. 5B). These results indicated that recombinant NRF2-siRNA could selectively modulate the expression of particular $\mathrm{ABC}$ transporters in human OS cells.

Recombinant NRF2-siRNA Molecule Is Effective to Enhance the Sensitivity of Human OS Cells to Doxorubicin, Cisplatin, and Sorafenib. Given the important role of NRF2 in chemosensitivity of cancer cells (Kim et al., 2008; Xu et al., 2014; Li et al., 2017), we defined how interfering NRF2 with biologic siRNA molecule would affect the chemosensitivity of human OS cells. The results showed that a small dose $(2 \mathrm{nM})$ of BERA/NRF2-siRNA significantly $(P<0.001$, two-way ANOVA) enhanced the sensitivity of both 143B and MG63 cells to doxorubicin, cisplatin, and sorafenib, as compared with MSA control (Fig. 6, A-C). The improved chemosensitivity was further demonstrated by the lower $\mathrm{EC}_{50}$ and "top" values in BERA/NRF2-siRNA-treated cells than corresponding controls (Fig. 6D). Together, these findings indicated that the interference of NRF2 pathways with biologic siRNA molecule could enhance the efficacy of doxorubicin, cisplatin, and sorafenib against OS cells.

\section{Discussion}

Understanding the functions of transcription factor NRF2 in the regulation of target gene expression and its potential as a therapeutic target depends upon the utilization of proper genetic (Singh et al., 2008; Fourtounis et al., 2012; Lu et al., 2016) and pharmacological (Sun et al., 2017) tools. Distinguished from synthetic siRNA molecules bearing extensive and a wide array of artificial modifications, which vary from different manufacturers and thus pose distinct structures, physicochemical properties, and biologic/pharmacological activities, the new recombinant siRNA agent produced in the present study using a novel 
A

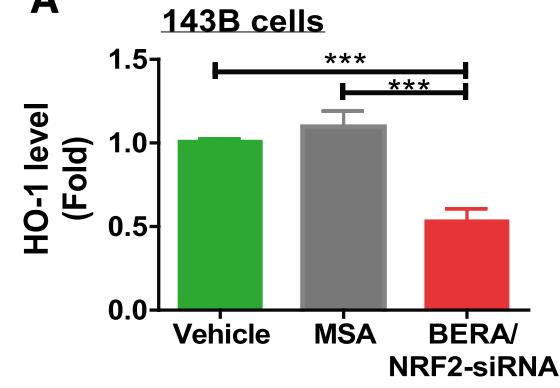

MG63 cells

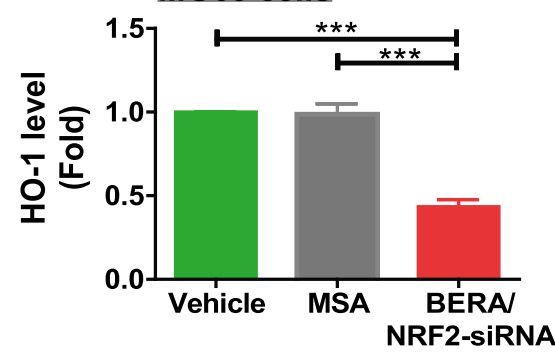

B
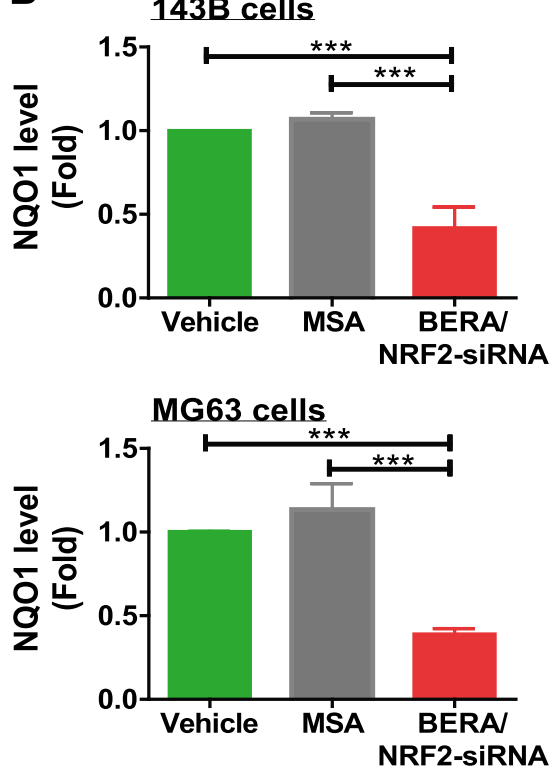

C $143 B$ cells

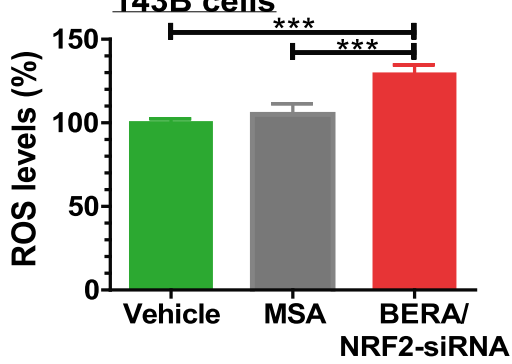

MG63 cells

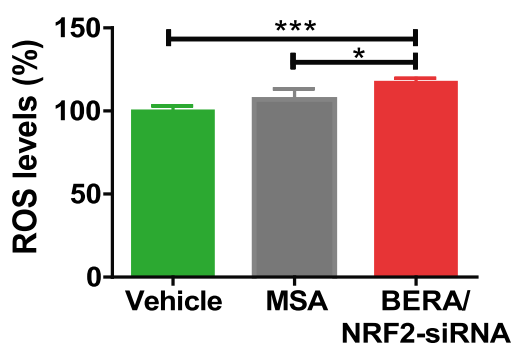

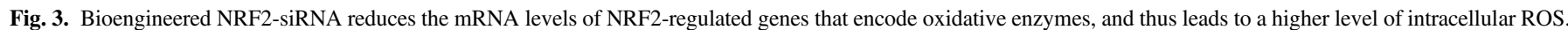

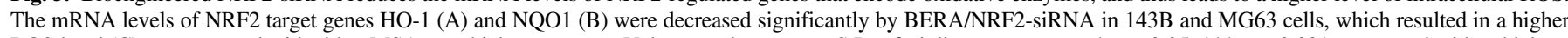

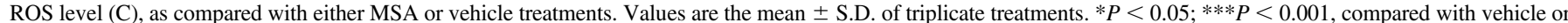
MSA controls (one-way ANOVA).

tRNA/pre-miR-34a-based ncRNA bioengineering technology (Chen et al., 2015) should better capture the properties of natural RNA molecules. Further comprehensive studies demonstrated a selective knockdown of NRF2 mRNA and protein levels within human OS 143B and MG63 cells, following the production of target NRF2-siRNA from BERA/NRF2-siRNA. This led to the interference of NRF2 signaling pathways, as manifested by remarkable changes of NRF2-regulated antioxidant enzymes and efflux transporters. Consequently, bioengineered NRF2-siRNA was effective to modulate intracellular ROS levels and sensitize OS cells to doxorubicin, cisplatin, and sorafenib.

NRF2 has been traditionally considered as a critical factor for cell defense and survival, given the fact that NRF2 governs the expression of many detoxification and antioxidant enzymes (Furfaro et al., 2016).
Indeed, NRF2 is sequestered by Kelch-like ECH-associated protein 1 (KEAP1) in cytoplasm under normal physiologic conditions. In response to endogenous and exogenous oxidative or electrophilic stress, NRF2 escapes from KEAP1 suppression, activates its target gene expression after being translocated into the nucleus, and consequently provides chemoprotection. However, NRF2-mediated cytoprotective mechanism is reprogrammed and magnified in a variety of cancer cells. Furthermore, activation of NRF2 facilitates tumor progression and enhances multidrug resistance of cancer cells (Jaramillo and Zhang, 2013; Huang et al., 2015; Furfaro et al., 2016). Therefore, a more complete understanding of NRF2 regulatory pathways in cancer cells is necessary, and our bioengineered NRF2-siRNA molecule, whose actions have been demonstrated in the current study, may be a valuable

A
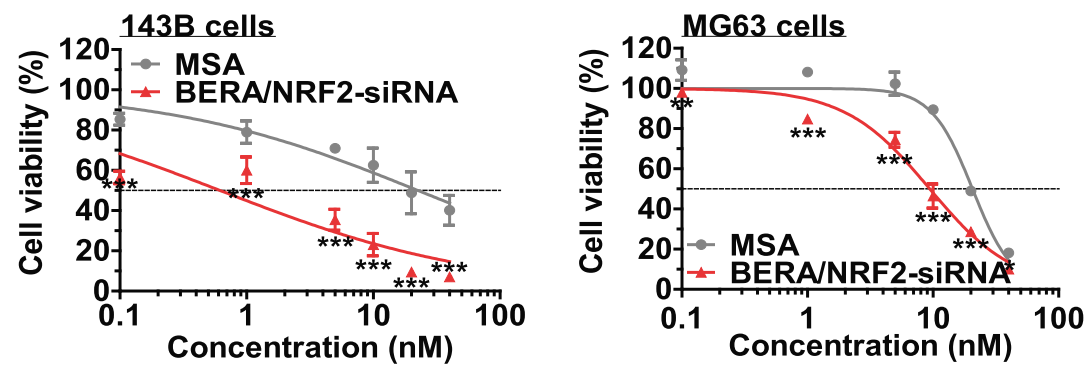

B

\begin{tabular}{|c|c|c|c|c|}
\hline \multirow[b]{2}{*}{ Cell line } & \multicolumn{2}{|c|}{ EC50 (nM) } & \multicolumn{2}{|c|}{ Hill Slope } \\
\hline & MSA & $\begin{array}{c}\text { BERA } \\
\text { NRF2-siRNA }\end{array}$ & MSA & $\begin{array}{c}\text { BERA } \\
\text { NRF2-siRNA }\end{array}$ \\
\hline 143B & $22.46 \pm 1.21$ & $0.62 \pm 1.38^{\star * *}$ & $-0.44 \pm 0.06$ & $-0.42 \pm 0.06$ \\
\hline MG63 & $20.61 \pm 1.05$ & $9.42 \pm 1.07^{* \star *}$ & $-2.67 \pm 0.33$ & $-1.28 \pm 0.12^{* * *}$ \\
\hline
\end{tabular}

Fig. 4. Bioengineered NRF2-siRNA inhibits human OS cell proliferation. (A) BERA/NRF2-siRNA significantly suppressed the growth of 143B and MG63 cells in a dose-dependent manner and to a greater degree than the control MSA (values are the mean \pm S.D. of triplicate treatments; two-way ANOVA with Bonferroni post-hoc tests: $P<0.001$ for drug treatment and dose; $* * * P<0.001 ; * * P<0.01 ; * P<0.05$ at indicated doses, compared with corresponding MSA control). (B) The estimated $\mathrm{EC}_{50}$ and Hill slope values for the inhibition of human OS cell proliferation by BERA/NRF2-siRNA and control MSA (*** $P<0.001$, compared with the MSA control; Student's $t$ test). 

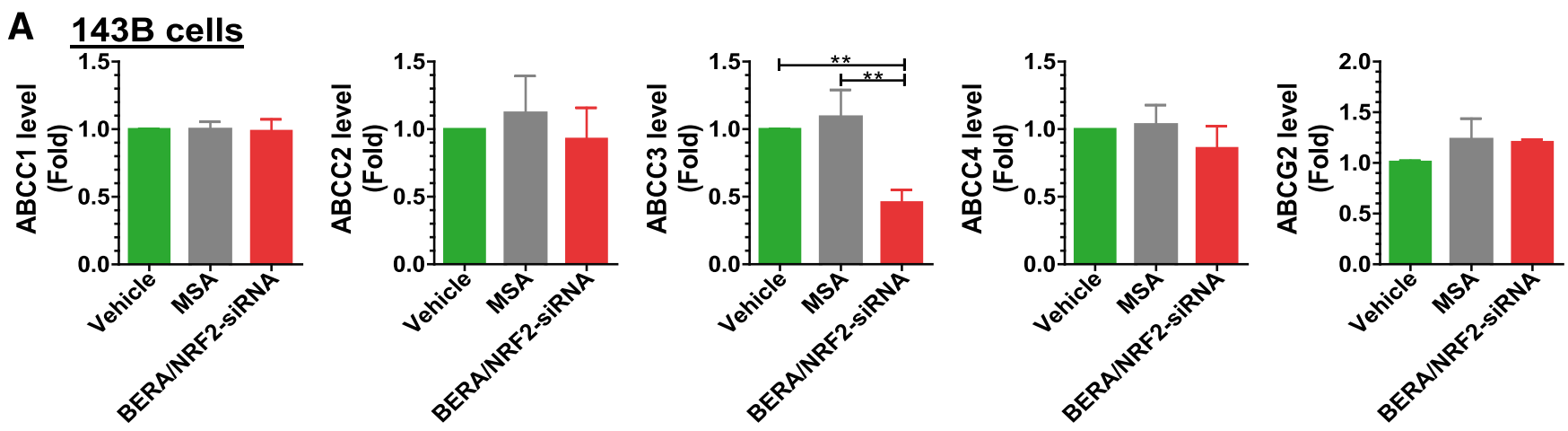

\section{B MG63 cells}
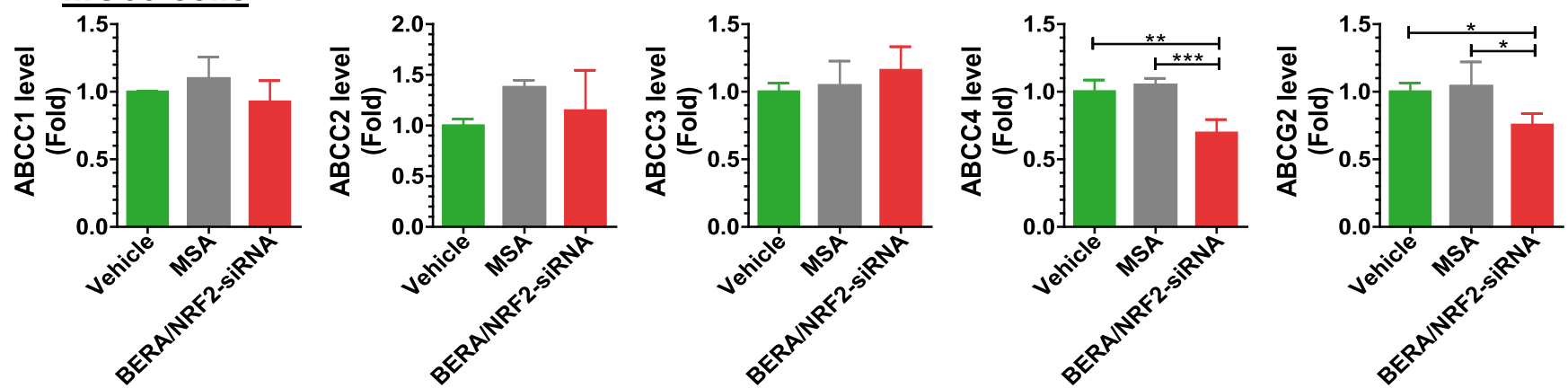

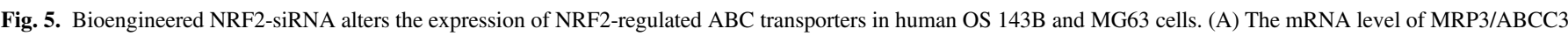

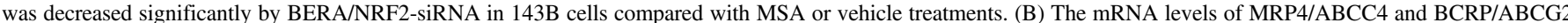

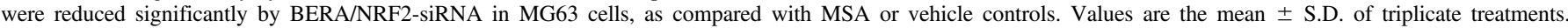
$* P<0.05 ; * * P<0.01 ; * * * P<0.001$, compared with vehicle or MSA treatments (one-way ANOVA).

addition to existing tools to interfere with the NRF2 pathway toward improved understandings.

NRF2 is indeed dysregulated in various types of tumors, including OS. One study (Park et al., 2012) revealed that NRF2 is positively expressed in many clinical OS specimens, which forecasts a poor prognostic outcome and correlates with worse disease-free survival. Similarly, another study (Zhang et al., 2016) identified nuclear expression rates 8-fold higher for NRF2 in OS tissues than normal peritumoral bone tissues, and positive NRF2 expression in OS patients is associated with a significantly lower 5-year survival rate. By contrast, the expression rate of KEAP1, the repressive partner of NRF2, is around 7-fold lower in OS tissues than normal peritumor samples; the 5-year survival rate is significantly higher in OS patients showing positive KEAP1 expression, as compared with those with negative expression (Zhang et al., 2016). In accordance with targeting NRF2 to control cancer (DeNicola et al., 2011), we observed a dose-dependent antiproliferative activity for bioengineered NRF2-siRNA molecule following the interference of NRF2 signaling pathways in OS cells. These findings should provide insight into developing a new strategy to inhibit NRF2 signaling for the treatment of OS.

The HO-1 enzyme is one of the main targets of NRF2 and a major effector of NRF2-dependent cell response to oxidative stress and xenobiotics (Teppner et al., 2016). A concurrent upregulation of NRF2 and HO-1 is obvious in many types of tumors and correlates well with tumor progression, resistance to therapy, and clinical outcomes (Furfaro et al., 2016). Our finding on the reduction of HO-1 and NQO1 levels in both 143B and MG63 cells demonstrated the consequent effects of knocking down NRF2 on NRF2-regulated gene expression by bioengineered NRF2-siRNA. Furthermore, a higher accumulation of intracellular ROS highlighted the impact of interfering NRF2/HO-1 axis on redox homeostasis (DeNicola et al., 2011; Teppner et al., 2016). In addition, the change of cellular ROS levels following the perturbation of NRF2/HO-1 axis should provide at least partial explanation for the antiproliferative activity of bioengineered NRF2-siRNA against OS cells, as well as the improved cell sensitivity to doxorubicin, cisplatin, and sorafenib.

Chemosensitivity of cancer cells also involves efflux ABC transporter-mediated mechanisms (Choi and $\mathrm{Yu}, 2014)$. In agreement with the role of NRF2 in drug resistance, NRF2 has been demonstrated to regulate the expression of many $\mathrm{ABC}$ transporters, including ABCB6, ABCC1-5, and ABCG2, which may differ due to the differences in types of cells with variable levels of expression and/or distinct regulatory pathways (Adachi et al., 2007; Aleksunes et al., 2008; Malhotra et al., 2010; Singh et al., 2010; Xu et al., 2010; Chorley et al., 2012; Canet et al., 2015; Jeong et al., 2015; Francois et al., 2017). Through interfering NRF2 with bioengineered NRF siRNA, the present study revealed a consequent reduction of ABCC3 expression in 143B cells as well as the suppression of ABCC4 and ABCG2 in MG63 cells, supporting the role of NRF2 in the regulation of $\mathrm{ABC}$ transporter expression. Our findings also agree with previous findings on possible differences in the regulation of distinct $\mathrm{ABC}$ transporters in different cells lines. For example, ABCC4 levels are significantly upregulated in HepG2 cells and human hepatocytes after the activation of NRF2 (Xu et al., 2010), whereas ABCG2 levels are readily reduced in lung and prostate cancer cells after the knocking down of NRF2 (Singh et al., 2010). In addition, consistent with previous findings of an increased chemosensitivity for lung cancer cells after the disruption of NRF2-ABCG2 cascade (Singh et al., 2010), treatment with bioengineered NRF2-siRNA sensitized OS cells to doxorubicin, cisplatin, and sorafenib. Given the facts that doxorubicin and sorafenib are substrates of $\mathrm{ABC}$ transporters 
A

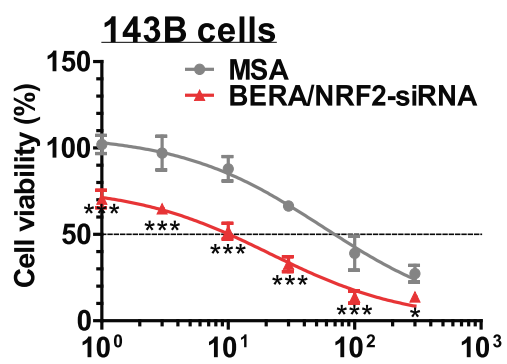

Doxorubicin concentration (nM)

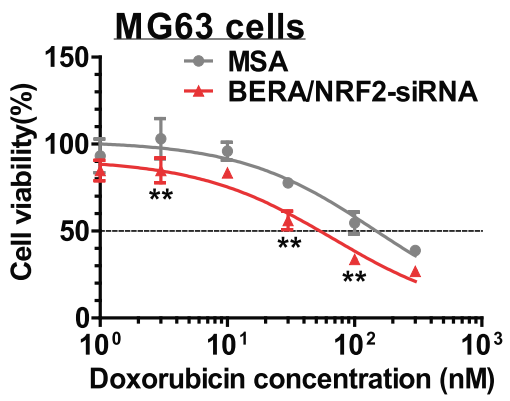

B
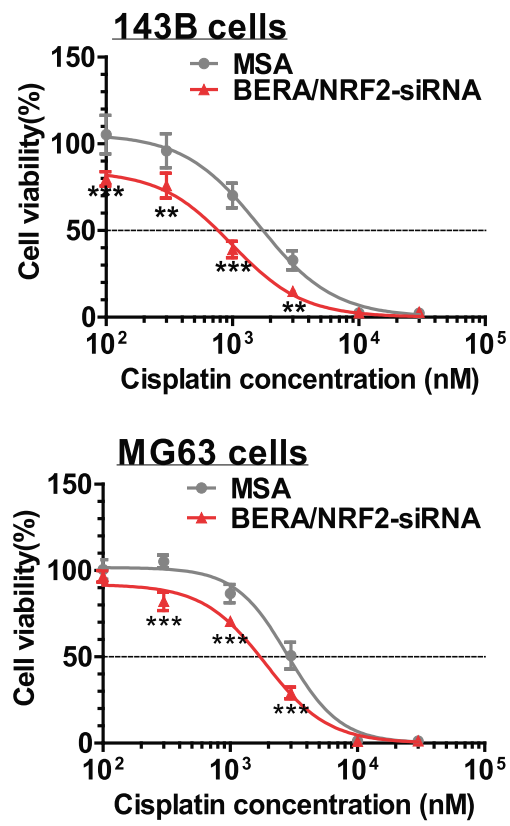

C
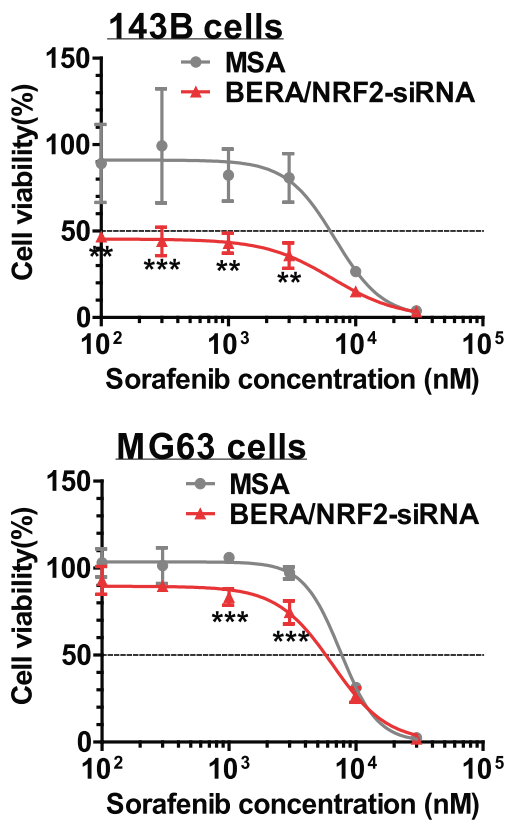

\begin{tabular}{|c|c|c|c|c|c|c|c|}
\hline \multirow[b]{2}{*}{ Drug } & \multirow[b]{2}{*}{$\begin{array}{l}\text { Cell } \\
\text { line }\end{array}$} & \multicolumn{2}{|c|}{ EC50 (nM) } & \multicolumn{2}{|c|}{ Hill Slope } & \multicolumn{2}{|c|}{ Top } \\
\hline & & MSA & $\begin{array}{c}\text { BERA/ } \\
\text { NRF2-siRNA }\end{array}$ & MSA & $\begin{array}{c}\text { BERA/ } \\
\text { NRF2-siRNA }\end{array}$ & MSA & $\begin{array}{c}\text { BERA/ } \\
\text { NRF2-siRNA }\end{array}$ \\
\hline \multirow{2}{*}{ Doxorubicin } & 143B & $57.41 \pm 1.23$ & $21.44 \pm 1.27^{* * *}$ & $-0.76 \pm 0.11$ & $-0.79 \pm 0.11$ & $107.8 \pm 5.5$ & $77.66 \pm 4.99^{* * *}$ \\
\hline & MG63 & $142.2 \pm$ & $67.59 \pm 1.24^{\star \star *}$ & $-0.82 \pm 0.16$ & $-0.81 \pm 0.13$ & $101.8 \pm 4.7$ & $91.37 \pm 4.77^{\star \star \star *}$ \\
\hline \multirow{2}{*}{ Cisplatin } & $143 B$ & $1620 \pm 1$ & $982.8 \pm 1.1^{\star \star \star *}$ & $-1.46 \pm 0.20$ & $-1.53 \pm 0.19$ & $105.7 \pm 4.1$ & $84.29 \pm 3.29^{\star \star *}$ \\
\hline & MG63 & $2832 \pm 1$ & $1894 \pm 1^{\star \star *}$ & $-2.10 \pm 0.26$ & $-1.76 \pm 0.17^{\star \star *}$ & $101.7 \pm 2.2$ & $91.97 \pm 2.15^{\star \star \star}$ \\
\hline \multirow{2}{*}{ Sorafenib } & $143 B$ & $6882 \pm 1$ & $6512 \pm 1^{* * *}$ & $-2.29 \pm 0.94$ & $-1.65 \pm 0.32^{* *}$ & $91.07 \pm 6.10$ & $45.39 \pm 1.83^{\text {***}}$ \\
\hline & MG63 & $7559 \pm 1$ & $6503 \pm 1^{* * *}$ & $-2.97 \pm 0.49$ & $-2.01 \pm 0.23^{* * *}$ & $103.6 \pm 1.8$ & $89.56 \pm 1.91^{* * *}$ \\
\hline
\end{tabular}

Fig. 6. BERA/NRF2-siRNA improves the chemosensitivity of human OS cells. BERA/NRF2-siRNA ( $2 \mathrm{nM})$ significantly sensitized $143 \mathrm{~B}$ and MG63 cells to doxorubicin (A), cisplatin (B), and sorafenib (C), respectively (values are the mean \pm S.D. of triplicate treatments; two-way ANOVA with Bonferroni post-hoc tests: $P<0.001$ for drug treatment and dose; $* * * P<0.001 ; * * P<0.01$ at indicated doses, compared with the same doses of MSA). (D) The estimated $\mathrm{EC}_{50}$, Hill slope, and top values for doxorubicin, cisplatin, and sorafenib cytotoxicity against BERA/NRF2-siRNA- and MSA-treated 143B and MG63 cells $(* * P<0.01 ; * * * P<0.001$, compared with MSA control; Student's $t$ test).

(Miyake et al., 1999; Zhou et al., 2001; Lagas et al., 2010; Agarwal et al., 2011) and cisplatin efficacy is highly related to ABCC2 expression [see review by Baiceanu et al. (2015)], the suppression of $\mathrm{ABC}$ transporter expression may offer further explanations for the enhanced chemosensitivity, other than the direct antiproliferative activity of NRF-siRNA.

In summary, we demonstrated a successful high-level production of a novel biologic NRF2-siRNA agent using our newly established ncRNA bioengineering technology. The bioengineered NRF2-siRNA molecule was effective to silence NRF2 expression in human cancer cells, which consequently reduced the expression of NRF2-regulated oxidative enzymes and led to higher intracellular ROS levels. In addition, knocking down NRF2 with bioengineered siRNA agent improved chemosensitivity of cancer cells, which was associated with the suppression of NRF2-regulated efflux ABC transporters. These findings not only support the intervention of NRF2 signaling pathways as a new therapeutic strategy to combat cancer but also point to a promising new direction for the development and use of biologic RNAi agents for drug disposition research as well as broad basic and translational studies.

\section{Authorship Contributions}

Participated in research design: A.-X. Yu, Li, A.-M. Yu, Tu, Ho, Jilek,

Duan, Zhang.

Conducted experiments: Li, Tu, Ho, Jilek, Duan, Zhang.

Contributed new reagents or analytic tools: A.-M. Yu, A.-X. Yu.

Performed data analysis: Li, A.-X. Yu, A.-M. Yu, Tu, Ho, Jilek, Duan, Zhang.

Wrote or contributed to the writing of the manuscript: A.-X. Yu, Li, A.-M. $\mathrm{Yu}, \mathrm{Tu}, \mathrm{Ho}$, Jilek, Duan, Zhang.

\section{References}

Adachi T, Nakagawa H, Chung I, Hagiya Y, Hoshijima K, Noguchi N, Kuo MT, and Ishikawa T (2007) Nrf2-dependent and -independent induction of ABC transporters $\mathrm{ABCC} 1, \mathrm{ABCC} 2$, and ABCG2 in HepG2 cells under oxidative stress. J Exp Ther Oncol 6:335-348.

Agarwal S, Sane R, Ohlfest JR, and Elmquist WF (2011) The role of the breast cancer resistance protein (ABCG2) in the distribution of sorafenib to the brain. J Pharmacol Exp Ther 336:223-233. Aleksunes LM, Slitt AL, Maher JM, Augustine LM, Goedken MJ, Chan JY, Cherrington NJ, Klaassen CD, and Manautou JE (2008) Induction of Mrp3 and Mrp4 transporters during acetaminophen hepatotoxicity is dependent on Nrf2. Toxicol Appl Pharmacol 226:74-83.

Baiceanu E, Crisan G, Loghin F, and Falson P (2015) Modulators of the human ABCC2: hope from natural sources? Future Med Chem 7:2041-2063.

Canet MJ, Merrell MD, Harder BG, Maher JM, Wu T, Lickteig AJ, Jackson JP, Zhang DD, Yamamoto M, and Cherrington NJ (2015) Identification of a functional antioxidant response element within the eighth intron of the human ABCC3 gene. Drug Metab Dispos 43:93-99. 
Chen N, Zhang R, Konishi T, and Wang J (2017) Upregulation of NRF2 through autophagy/ERK 1/2 ameliorates ionizing radiation induced cell death of human osteosarcoma U-2 OS. Mutat Res 813:10-17. Chen QX, Wang WP, Zeng S, Urayama S, and Yu AM (2015) A general approach to high-yield biosynthesis of chimeric RNAs bearing various types of functional small RNAs for broad applications. Nucleic Acids Res 43:3857-3869.

Choi YH and Yu AM (2014) ABC transporters in multidrug resistance and pharmacokinetics, and strategies for drug development. Curr Pharm Des 20:793-807.

Chorley BN, Campbell MR, Wang X, Karaca M, Sambandan D, Bangura F, Xue P, Pi J, Kleeberger SR, and Bell DA (2012) Identification of novel NRF2-regulated genes by ChIP-Seq influence on retinoid X receptor alpha. Nucleic Acids Res 40:7416-7429.

DeNicola GM, Karreth FA, Humpton TJ, Gopinathan A, Wei C, Frese K, Mangal D, Yu KH, Yeo CJ, Calhoun ES, et al. (2011) Oncogene-induced Nrf2 transcription promotes ROS detoxification and tumorigenesis. Nature 475:106-109.

Duan $\mathrm{Z}$ and $\mathrm{Yu}$ AM (2016) Bioengineered non-coding RNA agent (BERA) in action Bioengineered 7:411-417.

Fourtounis J, Wang IM, Mathieu MC, Claveau D, Loo T, Jackson AL, Peters MA, Therien AG, Boie Y, and Crackower MA (2012) Gene expression profiling following NRF2 and KEAP1 siRNA knockdown in human lung fibroblasts identifies CCL11/Eotaxin-1 as a novel NRF2 regulated gene. Respir Res 13:92.

Francois LN, Gorczyca L, Du J, Bircsak KM, Yen E, Wen X, Tu MJ, Yu AM, Illsley NP, Zamudio $S$, et al. (2017) Down-regulation of the placental BCRP/ABCG2 transporter in response to hypoxia signaling. Placenta 51:57-63.

Furfaro AL, Traverso N, Domenicotti C, Piras S, Moretta L, Marinari UM, Pronzato MA, and Nitti M (2016) The Nrf2/HO-1 axis in cancer cell growth and chemoresistance. Oxid Med Cell Longev 2016:1958174.

Ho PY and Yu AM (2016) Bioengineering of noncoding RNAs for research agents and therapeutics. Wiley Interdiscip Rev RNA 7:186-197.

Huang Y, Li W, Su ZY, and Kong AN (2015) The complexity of the Nrf2 pathway: beyond the antioxidant response. J Nutr Biochem 26:1401-1413.

Jaffe N (2009) Osteosarcoma: review of the past, impact on the future. The American experience. Cancer Treat Res 152:239-262.

Jaramillo MC and Zhang DD (2013) The emerging role of the Nrf2-Keap1 signaling pathway in cancer. Genes Dev 27:2179-2191.

Jeong HS, Ryoo IG, and Kwak MK (2015) Regulation of the expression of renal drug transporters in KEAP1-knockdown human tubular cells. Toxicol In Vitro 29:884-892.

Kansara M, Teng MW, Smyth MJ, and Thomas DM (2014) Translational biology of osteosarcoma Nat Rev Cancer 14:722-735.

Kim HR, Kim S, Kim EJ, Park JH, Yang SH, Jeong ET, Park C, Youn MJ, So HS, and Park R (2008) Suppression of Nrf2-driven heme oxygenase-1 enhances the chemosensitivity of lung cancer A549 cells toward cisplatin. Lung Cancer 60:47-56.

Koyani CN, Kitz K, Rossmann C, Bernhart E, Huber E, Trummer C, Windischhofer W, Sattler W, and Malle E (2016) Activation of the MAPK/Akt/Nrf2-Egr1/HO-1-GCLc axis protects MG-63 osteosarcoma cells against 15d-PGJ2-mediated cell death. Biochem Pharmacol 104:29-41.

Lagas JS, van Waterschoot RA, Sparidans RW, Wagenaar E, Beijnen JH, and Schinkel AH (2010) Breast cancer resistance protein and P-glycoprotein limit sorafenib brain accumulation. Mol Cancer Ther 9:319-326.

Lau A, Tian W, Whitman SA, and Zhang DD (2013) The predicted molecular weight of Nrf2: it is what it is not. Antioxid Redox Signal 18:91-93.

Li K, Ouyang L, He M, Luo M, Cai W, Tu Y, Pi R, and Liu A (2017) IDH1 R132H mutation regulates glioma chemosensitivity through Nrf2 pathway. Oncotarget 8:28865-28879.

Li MM, Addepalli B, Tu MJ, Chen QX, Wang WP, Limbach PA, LaSalle JM, Zeng S, Huang M, and Yu AM (2015) Chimeric microRNA-1291 biosynthesized efficiently in Escherichia coli is effective to reduce target gene expression in human carcinoma cells and improve chemosensitivity. Drug Metab Dispos 43:1129-1136.

Li MM, Wang WP, Wu WJ, Huang M, and Yu AM (2014) Rapid production of novel premicroRNA agent hsa-mir-27b in Escherichia coli using recombinant RNA technology for functional studies in mammalian cells. Drug Metab Dispos 42:1791-1795.

Lu C, Xu W, Zhang F, Shao J, and Zheng S (2016) Nrf2 knockdown attenuates the ameliorative effects of ligustrazine on hepatic fibrosis by targeting hepatic stellate cell transdifferentiation. Toxicology 365:35-47.
Luetke A, Meyers PA, Lewis I, and Juergens H (2014) Osteosarcoma treatment - where do we stand? A state of the art review. Cancer Treat Rev 40:523-532.

Malhotra D, Portales-Casamar E, Singh A, Srivastava S, Arenillas D, Happel C, Shyr C, Wakabayashi N, Kensler TW, Wasserman WW, et al. (2010) Global mapping of binding sites for Nrf2 identifies novel targets in cell survival response through ChIP-Seq profiling and network analysis. Nucleic Acids Res 38:5718-5734.

Miyake K, Mickley L, Litman T, Zhan Z, Robey R, Cristensen B, Brangi M, Greenberger L, Dean M, Fojo T, et al. (1999) Molecular cloning of cDNAs which are highly overexpressed in mitoxantrone-resistant cells: demonstration of homology to $\mathrm{ABC}$ transport genes. Cancer Res 59:8-13.

Park JY, Kim YW, and Park YK (2012) Nrf2 expression is associated with poor outcome in osteosarcoma. Pathology 44:617-621.

Rivera-Valentin RK, Zhu L, and Hughes DP (2015) Bone sarcomas in pediatrics: progress in our understanding of tumor biology and implications for therapy. Paediatr Drugs 17:257-271.

Singh A, Boldin-Adamsky S, Thimmulappa RK, Rath SK, Ashush H, Coulter J, Blackford A, Goodman SN, Bunz F, Watson WH, et al. (2008) RNAi-mediated silencing of nuclear factor erythroid-2-related factor 2 gene expression in non-small cell lung cancer inhibits tumor growth and increases efficacy of chemotherapy. Cancer Res 68:7975-7984.

Singh A, Wu H, Zhang P, Happel C, Ma J, and Biswal S (2010) Expression of ABCG2 (BCRP) is regulated by Nrf2 in cancer cells that confers side population and chemoresistance phenotype. Mol Cancer Ther 9:2365-2376.

Sun H, Zhu J, Lin H, Gu K, and Feng F (2017) Recent progress in the development of small molecule Nrf2 modulators: a patent review (2012-2016). Expert Opin Ther Pat 27:763-785.

Tang X, Yuan F, and Guo K (2014) Repair of radiation damage of U2OS osteosarcoma cells is related to DNA-dependent protein kinase catalytic subunit (DNA-PKcs) activity. Mol Cell Biochem 390:51-59.

Teppner M, Boess F, Ernst B, and Pähler A (2016) Biomarkers of flutamide-bioactivation and oxidative stress in vitro and in vivo. Drug Metab Dispos 44:560-569.

Wang WP, Ho PY, Chen QX, Addepalli B, Limbach PA, Li MM, Wu WJ, Jilek JL, Qiu JX, Zhang $\mathrm{HJ}$, et al. (2015) Bioengineering novel chimeric microRNA-34a for prodrug cancer therapy: high-yield expression and purification, and structural and functional characterization. $J$ Pharmacol Exp Ther 354:131-141.

Xu S, Weerachayaphorn J, Cai SY, Soroka CJ, and Boyer JL (2010) Aryl hydrocarbon receptor and NF-E2-related factor 2 are key regulators of human MRP4 expression. Am J Physiol Gastrointest Liver Physiol 299:G126-G135.

Xu X, Zhang Y, Li W, Miao H, Zhang H, Zhou Y, Li Z, You Q, Zhao L, and Guo Q (2014) Wogonin reverses multi-drug resistance of human myelogenous leukemia K562/A02 cells via downregulation of MRP1 expression by inhibiting Nrf2/ARE signaling pathway. Biochem Pharmacol 92:220-234.

Zhang J, Wang X, Wu W, Dang H, and Wang B (2016) Expression of the Nrf2 and Keap1 proteins and their clinical significance in osteosarcoma. Biochem Biophys Res Commun 473:42-46.

Zhao Y, Tu MJ, Wang WP, Qiu JX, Yu AX, and Yu AM (2016) Genetically engineered premicroRNA-34a prodrug suppresses orthotopic osteosarcoma xenograft tumor growth via the induction of apoptosis and cell cycle arrest. Sci Rep 6:26611.

Zhao Y, Tu MJ, Yu YF, Wang WP, Chen QX, Qiu JX, Yu AX, and Yu AM (2015) Combination therapy with bioengineered miR-34a prodrug and doxorubicin synergistically suppresses osteosarcoma growth. Biochem Pharmacol 98:602-613.

Zhou S, Schuetz JD, Bunting KD, Colapietro AM, Sampath J, Morris JJ, Lagutina I, Grosveld GC Osawa M, Nakauchi H, et al. (2001) The ABC transporter Bcrp1/ABCG2 is expressed in a wide variety of stem cells and is a molecular determinant of the side-population phenotype. Nat Med 7:1028-1034.

Address correspondence to: Dr. Ai-Xi Yu, Department of Orthopedics, Zhongnan Hospital of Wuhan University, Wuhan, Hubei 430071, China. E-mail: yuaixi@whu. edu.cn; or Ai-Ming Yu, Department of Biochemistry and Molecular Medicine, UC Davis School of Medicine, Sacramento, CA 95817. E-mail: aimyu@ucdavis.edu 